\title{
ROTATIONAL EXCITATION OF SMALL CARBON CHAINS BY COLLISIONS WITH HE IN THE ISM
}

\author{
A. Spielfiedel ${ }^{1}$, F. Dayou ${ }^{1}$, F. Dumouchel ${ }^{2}$, N. Feautrier ${ }^{1}$, F. Lique $^{2}$ \\ and M.-L. Senent ${ }^{3}$
}

\begin{abstract}
Modeling of molecular emission from interstellar clouds requires the calculation of rate coefficients for excitation by collision with the most abundant species. Based on new highly correlated potential energy surfaces, rotational excitation of $\mathrm{C}_{2} \mathrm{H}$ and $\mathrm{C}_{2} \mathrm{H}^{-}$radicals in collision with $\mathrm{He}$ are investigated. $\mathrm{C}_{2} \mathrm{H}$ fine and hyperfine structure is taken into account. As already found for other systems, rate coefficients for the $\mathrm{C}_{2} \mathrm{H}^{-}$ion are larger by a factor up to 3 compared to the neutral species.
\end{abstract}

\section{Introduction}

The $\mathrm{C}_{2} \mathrm{H}$ ethynyl molecule was first detected in the interstellar medium (ISM) in 1974 (Tucker 1974). $\mathrm{C}_{2} \mathrm{H}$ is one of the most abundant molecule in the ISM. It is a crucial intermediate in the interstellar chemistry leading to long chain carbon compounds (Padovani et al. 2009). Recently, emission in several high- $N$ rotational transitions was observed in the circumstellar envelope of IRC+10 216 with the IRAM $30 \mathrm{~m}$ and with Herschel/HIFI telescope. Modeling of these observations will strongly constrain the physical and chemical properties of this carbon-rich star (De Beck et al. 2012). Moreover, its ${ }^{2} \Sigma$ ground state gives rise to Zeeman splitting, so $\mathrm{C}_{2} \mathrm{H}$ was found to be a suitable molecule to determine magnetic fi elds (Bel \& Leroy 1998). On the other part, the discovery of carbon chain anions in interstellar and circumstellar media has initiated many theoretical and experimental works on these species. Although $\mathrm{C}_{2} \mathrm{H}^{-}$has been already observed (Brünken et al. 2007) in the laboratory, this species seems to have a very low abundance in the ISM.

\footnotetext{
1 LERMA - UMR 8112, Observatoire de Paris, 5 place Jules Janssen, Meudon, France

2 LOMC - UMR 6294, CNRS-Université du Havre, 25 rue Philippe Lebon, BP. 540, 76058 Le Havre, France

3 Departmento de Fisica y Quimica Teóricas, Instituto de Estructura de la Materia, CSIC, Calle Serrano 121, 28006 Madrid, Spain
} 

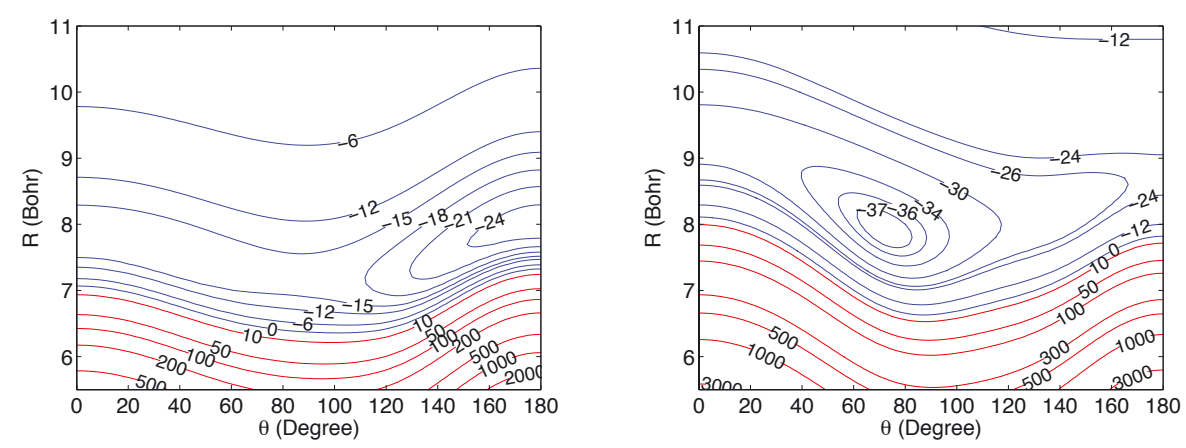

Fig. 1. Contour plot of the $\mathrm{C}_{2} \mathrm{H}-\mathrm{He}($ left $)$ and $\mathrm{C}_{2} \mathrm{H}^{-}$-He PESs as a function of $R$ and $\theta$. $\theta=0$ corresponds to colinear $\mathrm{He}-\mathrm{CCH}$ and $\mathrm{He}_{-} \mathrm{CCH}^{-}$.

Still, $\mathrm{C}_{2} \mathrm{H}^{-}$, is expected to be discovered with the new generation of sensitive astrophysical instruments such as ALMA.

Due to the low density of the interstellar medium, the populations of the rotational states of the molecules are not at local thermodynamical equilibrium. Thus modeling of molecular emission requires excitation calculations using radiative as well as collisional rate coefficients with the most abundant interstellar species He and $\mathrm{H}_{2}$. Collisional studies of $\mathrm{C}_{2} \mathrm{H}$ and $\mathrm{C}_{2} \mathrm{H}^{-}$with $\mathrm{He}$ at low temperatures were performed recently and in this proceeding (Spielfiedel et al. 2012; Dumouchel et al. 2012), we try to understand the particular behavior of negatively charged species during collisions and compare negative ion rate coefficients with those of neutral associated species.

\section{Potential energy surfaces and dynamics}

The calculation of the two Potential Energy Surfaces (PESs) was performed using quantum chemistry method using large basis sets, assuming rigid molecules for both the $\mathrm{C}_{2} \mathrm{H}$ and $\mathrm{C}_{2} \mathrm{H}^{-}$systems. Figure 1 displays the contour plot of the two 2D PESs as function of the distance between the He atom and the center of mass of $\mathrm{C}_{2} \mathrm{H}$ or $\mathrm{C}_{2} \mathrm{H}^{-}$and $\theta$, the angle between $R$ and the molecule axis. For these van der Waals systems, the global minimum of the interaction energy was found to be is $-25.54 \mathrm{~cm}^{-1}$ at $R=8.006 \mathrm{bohr}$ and $\theta=180^{\circ}$ and $-37.764 \mathrm{~cm}^{-1}$ at $R=7.91$ bohr, $\theta=72.38^{\circ}$ for $\mathrm{C}_{2} \mathrm{H}-\mathrm{He}$ and $\mathrm{C}_{2} \mathrm{H}^{-}-\mathrm{He}$ respectively. The two PESs exhibit important anisotropy for geometries close to the minimum, but a near-homonuclear symmetry for shorter internuclear distances.

\section{Temperature variation of $\mathrm{C}_{2} \mathrm{H}-\mathrm{He}$ and $\mathrm{C}_{2} \mathrm{H}^{-}-\mathrm{He}$ rate coefficients}

Cross sections among the 25 first fine structure $N j$ levels (where $N$ is the rotational angular momentum and $j$ the total angular momentum with $j=N+S$ ) of $\mathrm{C}_{2} \mathrm{H}$ and the 13 first rotational $N$ levels of $\mathrm{C}_{2} \mathrm{H}^{-}$were calculated in a full close 

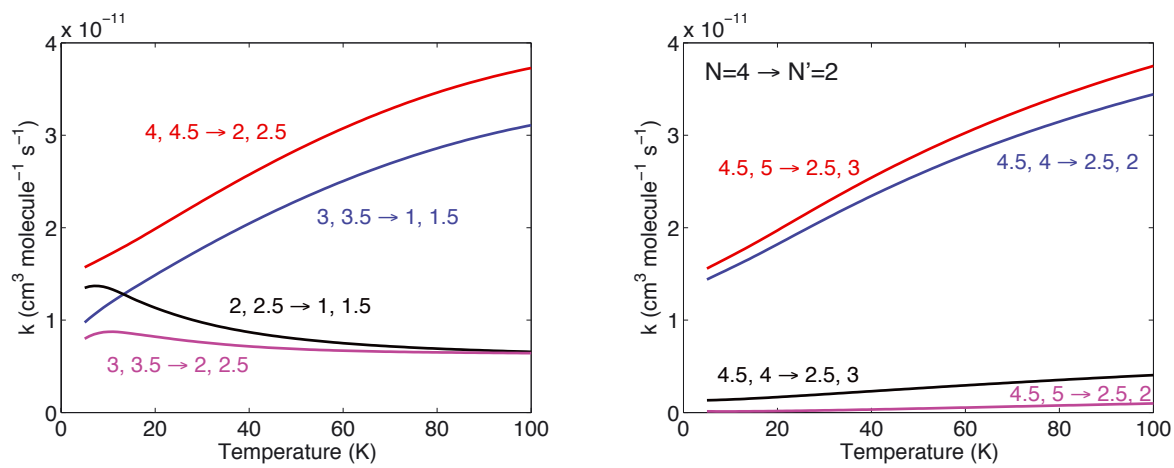

Fig. 2. Temperature variation of rate coefficients for $\mathrm{C}_{2} \mathrm{H}$ fine structure transitions $N, j \rightarrow N^{\prime}, j^{\prime}$ with $\Delta N=1,2$ (left) and hyperfine resolved transitions $N=4, j, F \rightarrow$ $N^{\prime}=2, j^{\prime}, F^{\prime}$ and $\Delta j=\Delta N$ transitions (right).

coupling approach for energies up to $800 \mathrm{~cm}^{-1}$. The exact electronic spin splitting of the $\mathrm{C}_{2} \mathrm{H}$ energy levels was taken into account. The recoupling technique introduced by Alexander \& Dagdigian (1985) was used to deduce the corresponding $\mathrm{C}_{2} \mathrm{H}$-He hyperfine $\left(N j F \rightarrow N^{\prime} j^{\prime} F^{\prime}\right.$, with $\left.F=j+I, I=1 / 2\right)$ cross sections. Rate coefficients for temperatures up to $100 \mathrm{~K}$ were derived from cross sections.

The variation with temperature of the de-excitation rate coefficients between fine structure levels of $\mathrm{C}_{2} \mathrm{H}$ (Fig. 2) exhibits a strong propensity for $\Delta N=2$ (due to near-homonuclear symmetry of the PES) and $\Delta j=\Delta N$ transitions. This last behaviour, predicted theoretically by Alexander et al. (1986), was found for other colliding systems between molecules in a ${ }^{2 S+1} \Sigma(S \neq 0)$ electronic state in collision with rare gas atoms. Considering transitions between hyperfine levels, the collisions exhibit propensity rules in favour of $\Delta F=\Delta j$ transitions for $\Delta j=\Delta N$ and no systematic trend is observed for the other transitions. Figure 3 displays the temperature variation of the $\mathrm{C}_{2} \mathrm{H}^{-}-\mathrm{He}$ de-excitation rate coefficients: apart from the lowest temperatures, those coefficients decrease slowly with $T$. The complete sets of (de)excitation rates coefficients are available online in the $\mathrm{LAMDA}^{1}$ and BASECOL $^{2}$ websites.

\section{Comparison between $\mathrm{C}_{2} \mathrm{H}-\mathrm{He}$ and $\mathrm{C}_{2} \mathrm{H}^{-}-\mathrm{He}$ rate coefficients}

For comparison, the $\mathrm{C}_{2} \mathrm{H}-\mathrm{He}$ rate coefficients between fine structure levels are averaged and summed over initial and final electronic spin quantum numbers respectively. The temperature dependence of the rate coefficients (Fig. 3) is different for the two colliding systems. This difference may be explained by considering the energy variation of the cross sections, dominated for $\mathrm{C}_{2} \mathrm{H}$-He collisions by large

\footnotetext{
${ }^{1}$ http://www.strw.leidenuniv.nl/ moldata/

${ }^{2}$ http://basecol.obspm.fr/
} 

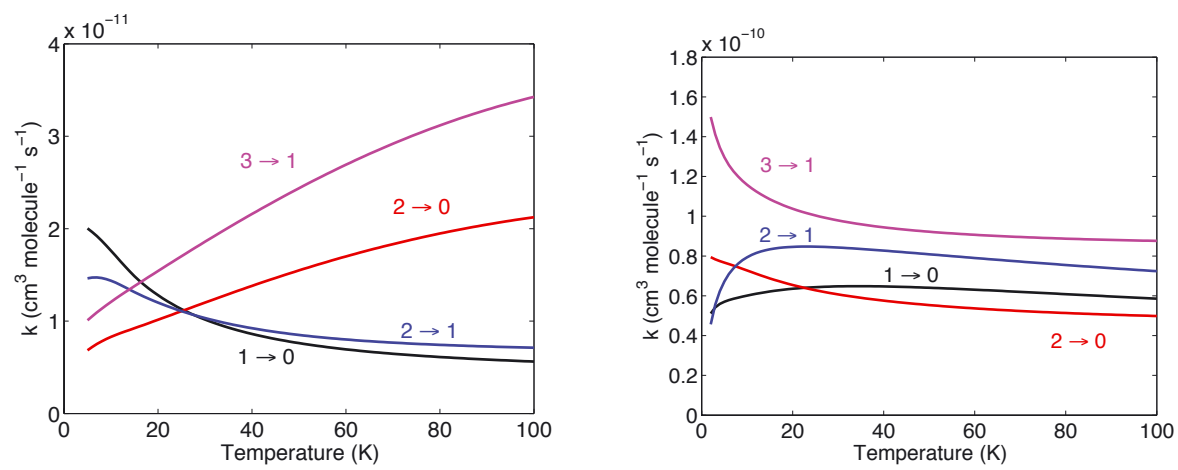

Fig. 3. Temperature variation of: de-excitation electronic spin averaged $\mathrm{C}_{2} \mathrm{H}-\mathrm{He} N \rightarrow N^{\prime}$ rate coefficients (left), $\mathrm{C}_{2} \mathrm{H}^{-}$-He de-excitation rate coefficients (right), with $\Delta N=2$.

resonances at low energies followed by a rapid decreasing for higher energies while the energy decreasing of the $\mathrm{C}_{2} \mathrm{H}^{-}$-He cross sections is much slower.

Rate coefficients for the $\mathrm{C}_{2} \mathrm{H}^{-}$anion are larger by factors up to 3 than the corresponding coefficients for the $\mathrm{C}_{2} \mathrm{H}$ neutral radical, depending on the transitions and the temperature considered. This difference could be partly due to the larger depth well for $\mathrm{C}_{2} \mathrm{H}^{-}$-He than for $\mathrm{C}_{2} \mathrm{H}-\mathrm{He}$ and also to the asymptotic dependence of the PESs which varies as $R^{-6}$ and $R^{-4}$ for $\mathrm{C}_{2} \mathrm{H}-\mathrm{He}$ and $\mathrm{C}_{2} \mathrm{H}^{-}$-He respectively (Dumouchel et al. 2012). Similar trend was found recently for $\mathrm{CN}^{-}$(Klos \& Lique 2011). Those results prove that rate coefficients for collisional excitation/deexcitation of neutral species cannot be used in place of the corresponding anion rate coefficients for modeling recent high resolution observations.

\section{References}

Alexander, M.H., \& Dagdigian, P.J., 1985, J. Chem. Phys., 83, 2191

Bel, N., \& Leroy, B., 1998, A\&A, 335, 1025

Brünken, S., Gottlieb, C.A., Gupta, H., \& McCarthy, M.C., 2007, A\&A, 464, L33

De Beck, R., Lombaert, R., Ahundez, M., et al., 2012, A\&A, 539, A108

Dumouchel, F., Spielfiedel, A., Senent, M.-L., et al., 2012, Chem. Phys. Lett., 533, 6

Klos, J., \& Lique, F., 2011, MNRAS, 418, 271

Padovani, M., Walmsley, C.M., Tafalla, et al., 2009, A\&A, 505, 1199

Spielfiedel, A., Feautrier, N., Najar, F., et al., 2012, MNRAS, 421, 1891

Tucker, K.D., Kutner, M.L., \& Thaddeus, P., 1974, ApJ, 193, L115 